\title{
Simultaneous Segmentation of the Left and Right Heart Ventricles in 3D Cine MR Images of Small Animals
}

\author{
J Schaerer $^{1}$, Y Rouchdy ${ }^{2}$, P Clarysse $^{1}$, B Hiba $^{3}$, \\ P Croisille $^{1}$, J Pousin ${ }^{2}$, IE Magnin ${ }^{1}$ \\ ${ }^{1}$ CREATIS CNRS UMR 5515, Inserm U 630, Lyon, France \\ ${ }^{2}$ Institut Camille Jordan UMR 5208, Lyon, France \\ ${ }^{3}$ ANIMAGE Platform, Rhône-Alpes Genopole, Lyon, France
}

\begin{abstract}
New high resolution image techniques allow to capture the anatomy and movement of the heart of small animals. The availability of these in vivo images can be very useful for medical research, however the amount of generated data for large animal studies makes manual analysis a very tedious task. To cope with the problem of automatic analysis of these images, we propose the use of the Deformable Elastic Template method to perform automatic segmentation of the ventricles. To adapt the method to the specificities of high-resolution MRI, several improvements are presented, including an image-context dependent scheme for more robust segmentation. Qualitative results show that our method is able to correctly retrieve the heart's contours in $3 D$.
\end{abstract}

\section{Introduction}

Small animal models are very useful for evaluating pharmacological and genetic therapies of cardiac pathologies such as myocardial ischemia. The heart function of small animals can now be studied using high resolution imaging techniques such as MRI. However, automatic extraction of the heart contours in these images is necessary to quickly measure vital heart functional parameters such as cavity volumes or ejection fraction. The goal of this study is to perform almost automatic $3 \mathrm{D}+$ time segmentation of the myocardium in MR images of the small animal.

Our method is based on the Deformable Elastic Template method introduced by Pham [1], and later improved by Rouchdy [2]. This method was originally developed for the analysis of human cardiac sequences.

Compared to human images, small animal MR images have a lower signal-to-noise (SNR) ratio and more movement artifacts. The use of a surface coil results in the presence of intensity inhomogeneities in the images. The Deformable Elastic Template method we previously proposed is well suited for these purposes, since it is quite robust to image noise and intensity inhomogeneity. However, the increased visibility of secondary structures such as arteries was a major problem we have to overcome, since it tends to confuse the segmentation process. In this article, we propose several improvements to adapt the method to high resolution images of the small animal.

\section{Material and method}

\subsection{Experimental data}

Mouse cine images were acquired on a $7 \mathrm{~T}$ magnetic resonance scanner with a whole body coil for RF excitation and a $15 \mathrm{~mm}$ surface coil for MR signal reception. An ECG-gated FLASH sequence was used to acquire shortaxis cine images with a $25 \mathrm{~mm}^{2} \mathrm{FOV}, 256 \times 256$ pixels, 1 mm slice thickness, 7/3.5 ms TR/TE, $64 \mathrm{KHz}$ bandwidth and 20 degrees flip-angles. Cine images (16 frames) were achieved for 7 slices, covering the entire LV. With a cardiac frequency of 450 b.p.m, the total acquisition time was of 20 minutes.

\subsection{Segmentation using the Deformable Elastic Template}

In order to ease the contour extraction process, one possibility is to use an a priori model of the object to be extracted that will be deformed iteratively to fit the image content. This approach is commonly known in image processing as deformable models. The proposed model presents the advantage of allowing the simultaneous extraction of both the endocardial and epicardial surfaces $[1,3]$. The concept, named Deformable Elastic Template, is a combination of :

- A topological and geometric model of the object to be segmented (see figure 2). In this context, this a priori model is a bi-cavity geometrical mesh that results from the 
manual segmentation of cardiac ventricles in a reference data set.

- A constitutive equation (elasticity) defining its behavior under applied external image forces that push the model's interfaces towards the image edges.

The equilibrium of the model is obtained through the minimization of the following global energy functional :

$$
E=E_{\text {elastic }}+E_{\text {data }}
$$

where $E_{\text {elastic }}$ represents the deformation energy of the model and $E_{d a t a}$ is the energy due to the external image forces.

\subsubsection{Internal energy term}

The model is considered as a Saint-Venant material elastic body [4]. Its elastic energy can be expressed as :

$$
E_{\text {elastic }}=\frac{1}{2} \int_{\Omega} \sigma^{T} \epsilon d \Omega
$$

where $\sigma$ and $\epsilon$ are the 3D strain and deformation vectors and $\Omega$ is the model domain. The material is considered to be isotropic and completely defined by its Young modulus and its Poisson coefficient. The energy can then be expressed as :

$$
E_{\text {elastic }}=\frac{1}{2} \int_{\Omega}(S u)^{T} D(S u) d \Omega
$$

where $S$ is a differential operator, $D$ is the elasticity matrix and $u$ is the displacement vector.

\subsubsection{External image energy}

The object boundaries are submitted to a 3D force field $t$. The expression of the external energy $E_{\text {data }}$ is :

$$
E_{\text {data }}(u)=-\int_{\Gamma} t . u d \Gamma
$$

with $\Gamma$ the border of the object domain $\Omega$. The force field is derived from the image edges, as will be shown in section 2.5.

\subsection{Global energy minimization}

The model is decomposed into tetrahedral elements. The segmentation is obtained through the minimization of the global energy functional $E$ using the Finite Element Method. A linear implementation of the model was first proposed by Pham. For this study, we used the non-linear implementation developed by Rouchdy [2], which has better convergence properties and numerical stability.

\subsection{Initial positioning of the model}

One of the primary problems of deformable model methods is their sensitivity to initialization. In order to tackle this problem, we used an automatic affine registration method based on the minimization of a combined gradient-based and mutual information functional [3].

\subsection{Force field computation}

The quality of the force field is essential for the model to satisfactorily converge to the data. The force field used in this study was computed in the following way : several image filtering techniques were applied to the image, in order to eliminate most artifacts and small features unnecessary for segmenting the heart (see section 2.5.1). Edge detection was then performed using the Canny algorithm. The resulting edges were then blurred with a Gaussian kernel. The gradient of the resulting image is a vector field that can be used as a force field. However, to further improve the process, we used Gradient Vector Flow (GVF) diffusion [5]. The GVF method has been shown to improve the ability of the model to converge to boundary concavities, and to increase the capture range of the force field. In order to increase it even further, we normalized the resulting force field, that is, we set the norms of all vector with a norm over a certain threshold to 1 without altering the vector directions.

\subsubsection{Image data preprocessing}

High resolution MR images have a relatively low SNR. Furthermore, the abundance of small features in the images (papillary muscles, coronaries, etc.) can lead to local minima of the model energy, leading to inaccurate segmentation. Both problems need to be addressed before the image edges can be extracted. We applied morphological opening for removing small features and noise while preserving strong edges. The result of the filter with a spherical structuring element can be seen in figure 1 .

\subsubsection{Multiresolution strategy}

The shape of the heart can vary a lot between individuals and between different phases of the heart cycle. The affine registration step is designed to adapt the model to the first image, but since it has few parameters, the registered model can still be far from the image borders. To overcome this problem, we used a multiresolution scheme : the model deformation process starts with a very low resolution image, to place the model close to the heart contours. The image resolution is then gradually increased for the model ton accurately converge towards the heart's contours. 


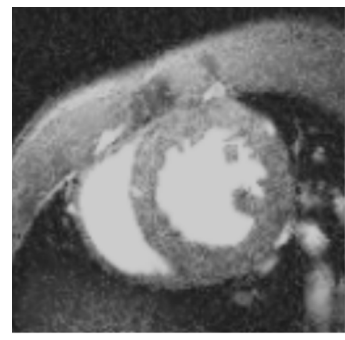

Original image

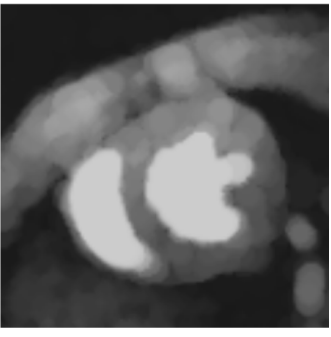

Filtered image
Figure 1. Morphological filtering (opening with a spherical structuring element with a radius of four pixels). Notice how small details and noise are removed by the filter.

To construct the multiresolution pyramid, images were recursively filtered with a Gaussian kernel and downsampled.

\subsection{Context-dependent application of the forces}

One advantage of an edge detection based force field is that it only depends on the presence of high gradients in the image. It does not depend on absolute gray value or even the sign of the gradients. The same scheme can thus be used on many different MRI sequences. On the other hand, it is impossible to distinguish between edges. In the case of the heart, the presence of very close edges (both sides of the ventricular walls) leads to problems with the deformable model. If the initialization is not good enough, one edge of the model can converge towards the wrong edge in the image, leading to a local minima in model energy (see figure 5 for an illustration). To address this problem, we introduced gradient sign information in the segmentation process. While this limits the generality of the method, it allows to distinguish the edges and allows for much more robust segmentation.

A smooth gradient of the 3D image was computed. During the deformation process, the normal of the model was compared with the gradient map : if the scalar product is negative, the force is considered to lead to the wrong edge and is not applied to the model. Using this method, a force is always applied at least on one side of the ventricular wall. The elasticity of the model guides the other side to the correct interface.

\section{Results}

Four 4D MR sequences corresponding to 4 different mice were processed using our methods. The parameters for the model were a Young modulus of $10 \mathrm{~Pa}$ for the LV and $40 \mathrm{~Pa}$ for the RV, and a Poisson coefficient of 0 for the whole model. We used 4 resolution levels, with 25 itera-

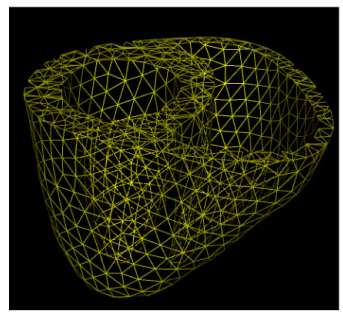

(a)

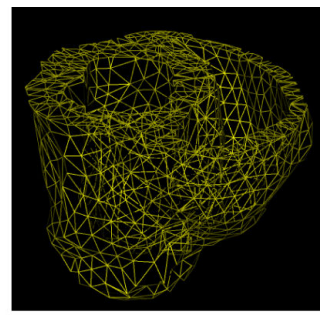

(b)
Figure 2. (a) Original model and (b) Deformed model
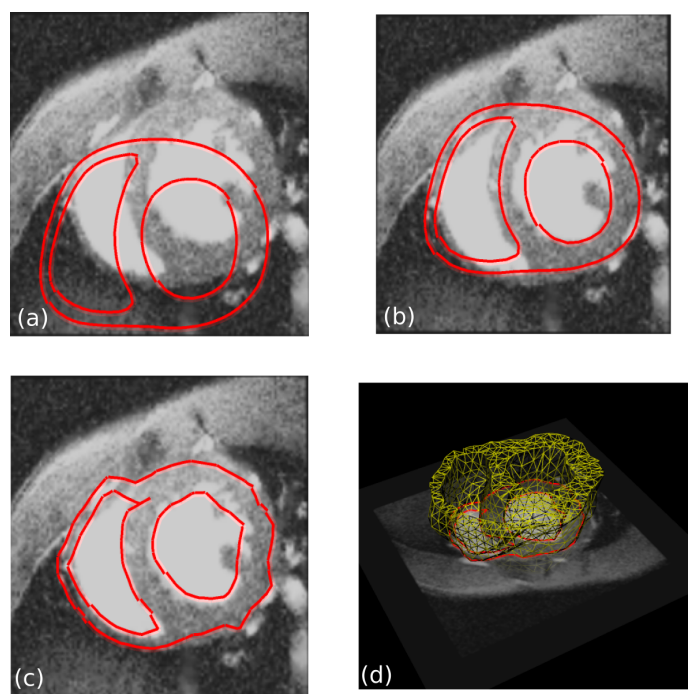

Figure 3. Segmentation process on mouse dataset \#1 : (a) Initial positioning, (b) After affine registration, (c) After deformation, (d) final result with mesh and image

tions for each of them. Results show that although we are still experiencing a few specific problems, our method was able to correctly locate the heart in the images and retrieve its contours.

\subsection{Qualitative results}

Figure 3 illustrates the process of the segmentation on one short-axis slice. Figure 2 shows the results of the deformation on the $3 \mathrm{D}$ mesh on the same $3 \mathrm{D}$ image.

\subsection{Effect of the non-linear elastic model}

We found that the non-linear model provides better numerical stability and convergence properties, especially under great deformations. Figure 4 illustrates the difference between meshes deformed with both schemes. While the shape is approximately the same, the quality of the mesh is much better in the non-linear case. 

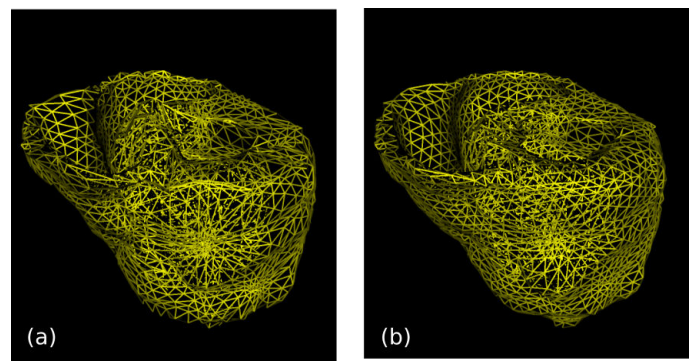

Figure 4. Linear vs. non-linear DET : (a) linear, and (b) non-linear.
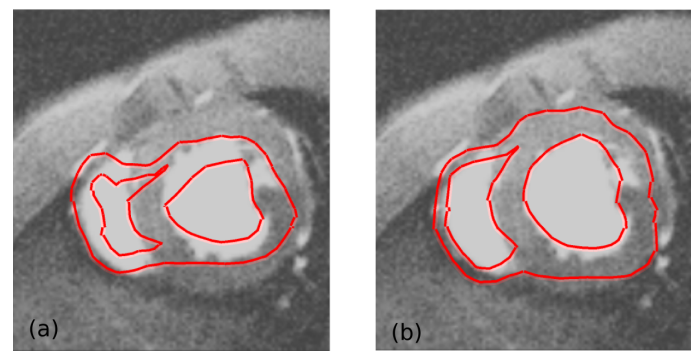

Figure 5. Effect of context-dependent application of the forces, illustrated on a medium slice of the whole 3D image : (a) Uniform application of the forces and (b) Gradient dependent application. Notice how the boundaries of the model converge towards the wrong edges when using the original scheme.

\subsection{Effect of context-dependent applica- tion of the forces}

Figure 5 illustrates the effect of the new scheme. Results shows that it mostly solves the problem of convergence towards the wrong boundaries, even if the initialization is far from the contour. The new scheme is thus much more robust to potential initial registration inaccuracies.

\subsection{Volume variation curves}

One direct application of segmentation is the automatic extraction of the cavity volumes. Once the contours are extracted, it is easy to compute the enclosed volumes. Figure 6 shows an example of volume variation curves obtained from automatic segmentation. While the overall variation pattern is good, remaining issues in segmentation during the early diasolic phase prevent us from giving more precise values.

\section{Discussion and conclusions}

The proposed method was able to retrieve the heart contours in most cases, enabling the computation of volume variation curves. Manual interaction and correction of the

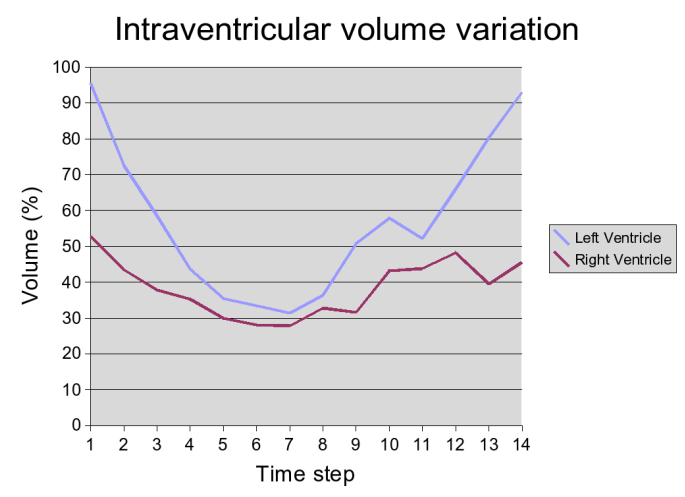

Figure 6. Estimated volume variation of the ventricles during the heart cycle (mouse dataset \#1)

results would however still be needed to use the method in routine experiments. Remaining problems include inaccuracies in the segmentation of the pericardium due to the presence of numerous anatomical structures near the heart, and errors during the early diastolic phase due to motion artifacts.

Future work will include the addition of a time constraint on the model to further improve segmentation accuracy over the cardiac cycle. Larger scale experiments, including quantitative evaluation of segmentation accuracy will also be needed to fully validate the method.

\section{References}

[1] Pham Q, Vincent F, Clarysse P, Croisille P, Magnin I. A FEM-based deformable model for the 3D segmentation and tracking of the heart in cardiac MRI. In Proceedings of the 2nd International Symposium on Image and Signal Processing and Analysis (ISPA 2001). 2001; 250-254.

[2] Rouchdy Y. Segmentation of the heart ventricles using 3D deformable elastic models. In Oberwolfach Seminar: Shape Optimization. 2004;

[3] Mäkelä T, Pham QC, Clarysse P, Nenonen J, Lötjönen J, Sipilä O, Hänninen H, Lauerma K, Knuuti J, Katila T, Magnin IE. A 3-D model-based registration approach for the PET, MR and MCG cardiac data fusion. Medical Image Analysis 2003;7:377-389.

[4] Ciarlet PG. Mathematical Elasticity Volume I: ThreeDimensional Elasticity. North-Holland, Amsterdam, 1988.

[5] Xu C, Prince JL. Snakes, shapes, and gradient vector flow. IEEE Transactions on Image Processing 1998;7:359-369.

Address for correspondence:

Joël Schaerer

CREATIS CNRS UMR 5515, Inserm U 630

INSA de Lyon 7, avenue Jean Capelle 69621 Villeurbanne Cedex joel.schaerer@creatis.insa-lyon.fr 